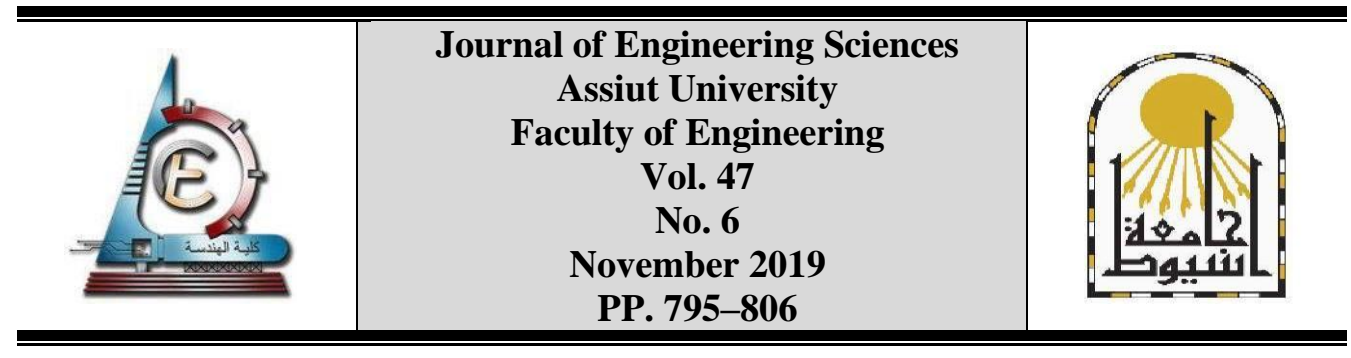

\title{
EARLY WARNING AND WATER QUALITY, LOW-COST IOT BASED MONITORING SYSTEM
}

\author{
Noha Kamal $^{*}, 1$, Abdallah Hammad ${ }^{2}$, Talaat Salem ${ }^{3}$, Mohie Omar ${ }^{4}$ \\ ${ }^{1,3,4}$ Nile Research Institute (NRI), National Water Research Center (NWRC), Egypt. \\ ${ }^{2}$ Depart. of Electrical Eng., Faculty of Engineering at Shoubra, Benha University, Egypt.
}

Received 24 March 2019; Accepted 1 July 2019

\begin{abstract}
In Egypt, the current water quality monitoring program involves thorough physio-chemical and biological analyses. However, there is still a lack of real-time water quality data that influences the urgent decision making process. The field acquisition of such data has still been costly, lengthy and laborious. Therefore, this paper aims to present a low-cost and labor-saving Early Warning Framework (EWF) for water quality monitoring of the River Nile based on the Internet of Things (IOT). A newly developed Prototype was introduced to monitor the in-situ water quality parameters; $\mathrm{pH}$, turbidity and temperature at a pilot location along the River Nile within Egypt. The same parameters were also monitored using the current state-of-the-art multi-probe EXO.Then, both sets of data measurements were sent to a real-time monitoring control center for comparison and calibration. The comparison results revealed that there is no significant difference between the two measurements according to a statistical analysis done using the Minitab 16 statistical model. The Root Mean Squared Error (RMSE) values showed that the error percentages were accepted for the three monitored parameters $(0.19$ for $\mathrm{pH}, 0.056$ for temperature, and 0.52 for turbidity). Moreover, the overall cost of the developed Prototype including sensors, raspberry Pi and all other expenses was found to be only $197 \$$ as compared to 11,130 \$ when using EXO. Accordingly, it could be concluded that the developed Prototype can provide a low-cost early warning system for water quality monitoring. Finally, it is strongly recommended to install developed real-time water quality monitoring stations as economic wireless hotspots at a number of strategic sites along the River Nile within Egypt.
\end{abstract}

Keywords: Water quality monitoring, Internet of Things, Raspberry PI, EXO.

\section{Introduction}

Traditional methods of water quality monitoring involve the manual collection of water samples at various locations and different times, followed by laboratory analyses in order to characterize the water quality. However, such methods are still insufficient to monitor the emergency pollution incidents on time. In Egypt, the Nile Research Institute (NRI) has conducted different water quality monitoring programs in collaboration with different

\footnotetext{
* Corresponding author.

E-mail address: noha_kamal2002@hotmail.com,noha_kamal2002@nri-eg.org
} 
agencies since 1976 in order to achieve several objectives. Since 2002, NRI has been carrying out the National Water Quality Program. This program includes collecting water samples from 4 sites at Lake Nasser, 25 sites along the River Nile and its two branches; Damietta and Rosetta, 9 irrigation canals and 2 rayahs and 29 point sources of pollution of agricultural drains at the points pouring into the river. The water samples collected from different water bodies are analyzed for physio-chemical and microbiological parameters during different seasons. Some water samples need to be analyzed immediately either in-situ or in the laboratory to get the right values. Such samples include Dissolved Oxygen (DO), Temperature, pH, Electrical Conductivity (EC), Total Alkalinity (TA) and Turbidity (Turb).

Although, the currently applied methodology at NRI provides a thorough analysis including chemical and biological agents, it, still, has several drawbacks namely; i) the lack of real-time water quality data that is indispensible for making quick and optimal decisions for public health protection. In fact, there is a wide time gap between sampling and detection of contamination. ii) the poor spatio-temporal coverage (a small number of locations are sampled), and iii) it is laborious and costly (labor, operation, and equipment). Therefore, there is a dire need for continuous on-line water quality monitoring with efficient spatiotemporal resolution. This paper proposes a water pollution warning system on the basis of the Internet of Things (IoT) using automatic water quality monitoring technology.

The IoT technique is used in different areas of research for monitoring, collecting and analyzing data from remote locations. Due to the increase in the amount of data, monitoring systems are becoming important factors in decision making. Internet of Things (IoT)-based sensors can be considered a solution to provide efficient water quality monitoring [1]. The IoT refers to a new kind of world where almost all the devices and appliances that we use are connected to a network. It can be used collaboratively to fulfil complex tasks that require a high degree of intelligence. For this intelligence and interconnection, IoT devices are equipped with embedded sensors, actuators, processors, and transceivers [2]. Currently, the Internet of Things (IoT) have an essential influence on all elements of human life. IoT as a prevalent phenomenon is transforming daily life by the usage of the smart features of Wireless Sensor Network (WSN) technologies [3].

The IoT architecture consists of different layers as shown in Figure 1, namely:

1) The perception layer is the physical layer which has sensors. In this study, it is related to only three different water quality parameters $(\mathrm{pH}$, Turbidity, and Temperature);

The transport layer is the networking layer. It transmits the sensor data from the perception layer to the processing layer and vice versa through networks such as Wi-Fi, 3G, LAN, Bluetooth, and GPRS;

2) The processing layer is also known as the middleware layer which stores, analyzes, and processes huge amounts of data coming from the transport layer. It can manage and provide diverse sets of services. It employs many technologies such as data bases, cloud computing, and big data processing modules. In the developed Prototype system, cloud computing technology is used to monitor data on the internet and make the system a user-friendly web browser application. In this way, the system will be low cost, faster, more efficient, real time and user friendly. Accordingly, the aim of the proposed system can be achieved; and the application layer hosts an alert system that can signal alert messages of water quality data to the decision maker. The administrators who have the privilege to access the 
developed website and mobile application that consist of real time visualizations of the system are responsible for delivering application specific services. They define various applications (smart homes, smart cities, and smart health) in which the IoT can be deployed. The developed system uses a website and a mobile application that can monitor the measuring process of water quality parameters remotely.

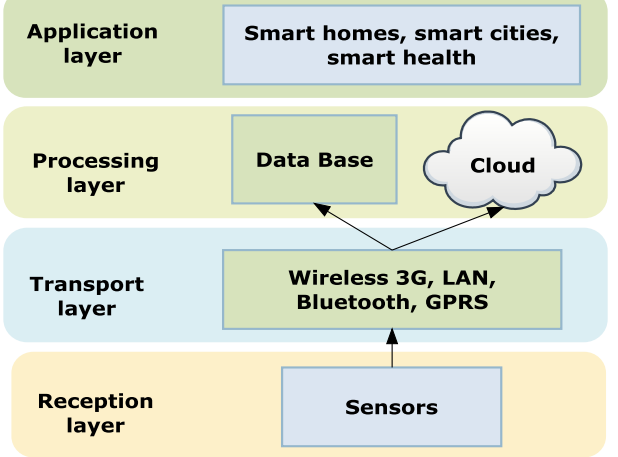

Fig. 1. IoT architecture

The IoT system is a basis for linking things, sensors and other smart technologies. It is known as Internet's advanced version [3]. It is an extension of the Internet. It allows objects to be remotely sensed and controled via network infrastructure. Thus, it creates opportunities of more integration of the physical world into a computer based system [4]. IoT gives an immediate access to data about physical objects and leads to highly efficient productive and innovative services. Cloud computing is a large-scale, low cost processing unit which is based on the IP; a connection for calculation and storage. In China, an example of water pollution warning framework was given on the basis of the IoT with a view to improving the speed of the pollution warning response in Beijing [4]. In addition, Fiji Islands which are located in the vast Pacific Ocean, the technology of IoT and RS were used to improve water quality monitoring and data collection [5].

Water scarcity and water contamination has been one of the major issues faced by the society around the world. In many developing countries like India, the main focus is given to industrialization and urbanization. But this has caused an increased water usage and water contamination, G. S. Menon, et al. design a Wireless Sensor node which contained several sensors. They highlighted on how a real time monitoring system can be made generic, using Network Virtualization. Also, they used new technologies Internet of Things (IoT) to make it advanced [6]. In addition,. K. Thiyagarajan, , et al. designed an intelligent IoT based Water Quality Monitoring system due to the rapidly increasing population in India, they monitored water quality automatically [7]. Brinda Das, and P.C.Jain implemented a real-time water quality monitoring system using Internet of Things, it can keep a strict check on the pollution of the water resources and be able to provide an environment for safe drinking water [8]. By 2020 , it is said that 50 billion 'things' will be linked together to the Internet [9].

In Egypt, few trials have been directing the IoT systems to water quality monitoring. The Water Quality Management Unit at the Ministry of Water Resources and Irrigation (MWRI) connected four automatic nodes to the central office. Each node has three sensors measuring $\mathrm{pH}, \mathrm{DO}$ and EC. The water quality data are transferred from the four nodes to the main station at the building of MWRI by GSM system. The network in Egypt has been stopped because of the high costs of labor, operation, and maintenance. The Telemetry 
Unit at the MWRI has installed a wireless system to monitor the water level at Lake Nasser. The system has been transferring data successfully by satellite. However, it has never been used in water quality monitoring along the River Nile.

This research basically aims to:

1) introduce a low cost and labor-saving Early Warning Framework (EWF) based on IoT at a pilot location for monitoring the water quality of the River Nile; and

2) modify and upgrade the current measurement system based on EXO so that it can instantaneously transfer the water quality parameters of the River Nile at a lower cost.

\section{Methodology}

This paper introduces an example of EWF to water quality monitoring of the River Nile within Egypt.The water pollution warning system is divided into three parts:

a. Sensing Terminal: It is the deployment of the automatic water quality pollution monitoring sensors to collect real-time data.

b. Transfer Network: It is the data information implementation, transfers the data based on IoT wireless network transmission.

c. Application Development: With these application systems, early warning information of water pollution will realize the high-speed transmission between substations units and superior station.

In order to achieve the research goal, some basic steps have to be done as follows:

Developing a low cost EWF for water quality.

- Upgrading the existing water quality monitoring system at NRI.

- Developing a dynamic website and mobile application.

- Analyzing the data by statistical model Minitab16.

- Analyzing the total cost.

\subsection{Development of a low cost early warning framework for water quality}

The current experiment installs a pilot sensing terminal node as the first part of EWF. The node is installed at the Nile Research Institute (NRI) site, Delta Barrage. The node location is at longitude $31^{\circ} 07^{\prime} 24^{\prime \prime} \mathrm{E}$ and latitude $30^{\circ} 11^{\prime} 51^{\prime \prime} \mathrm{N}$ at the western bank of the River Nile as shown in Figure 2. At this node, two types of sensors are used for water quality analyses. Prototype sensors which have been developed by the authors and NRI measuring unit based on EXO sensors which have been upgraded. Using these sensors, the collected data are sent to the main station at the main building of NRI via a wireless network channel. Figure 3 shows the block diagram of the developed prototype system. It can be noted that the perception layer consists of three sensors corresponding to three different water quality parameters $(\mathrm{pH}$, Turbidity, and Temperature). The main specifictions of $\mathrm{pH}$ sensor E-201 are Silver/Silver chloride reference electrode, operating temperature: $1^{\circ} \mathrm{C}-99^{\circ} \mathrm{C}$, max depth $60 \mathrm{M}$, and BNC connector. In addition, the turbidity sensor (LGZD Sensor V1.1) specifications are 5 VDC Supply Voltage, 30mA (MAX), Response time: $<500 \mathrm{~ms}$, output Analog (0-4.5V), and operating temperature: $-30{ }^{\circ} \mathrm{C}$ to 80 ${ }^{\circ} \mathrm{C}$. The tempreatuer sensor DS18B20 specifications are 12-bit Celsius temperature measurements, measures temperatures from $-55^{\circ} \mathrm{C}$ to $+125^{\circ} \mathrm{C}, \pm 0.5^{\circ} \mathrm{C}$ accuracy, and Power supply range is $3.0 \mathrm{~V}$ to $5.5 \mathrm{~V}$. 
In the transport layer, there are well known communication technologies such as Wi-Fi, Bluetooth, ZigBee and 2G/3G/4G cellular, and GSM/GPRS. The choice is a compromise between the right combination of power, coverage, data rate, and cost. Wi-Fi is a form of low-power wireless communication used by many electronic devices. The readings taken from the $\mathrm{pH}$ sensor which contains two electrodes, a sensor electrode and a reference electrode. They are in form of glass tubes one contains PH 7 buffer and other contains potassium chloride solution. Overall working principle of $\mathrm{pH}$ sensor depends on the exchange of ions from sample solution to inner solution (PH 7 buffer). Ph sensor interfaced with an analog to digital converter (mcp3008) to convert the measured analog voltage to the SPI protocol. The turbidity sensor which is operates on the principle that when light is passed through a sample of water, the amount of light transmitted through the sample is dependent on the amount of soil in the water. As the soil level increases, the amount of transmitted light decreases. It is also interfaced with the same A/D converter mcp3008. The mcp3008 alternates between two sensors as a slave to get a reading from each sensor at a time. In addition, the temperature sensor is a digital sensor interfaced directly to the raspberry Pi through one wire protocol. These sensed values are collected and processed by the raspberry Pi3 to make them suitable for reading and analysis. In the Transport layer, the processed values are sent to the cloud by using $\mathrm{Wi}-\mathrm{Fi}$. In the processing layer, a cloud computing technology is used to monitor the data on the internet. Eventually, in the Application layer, the processed data is monitored through a browser application and mobile application using a special username and password for each geographical area.

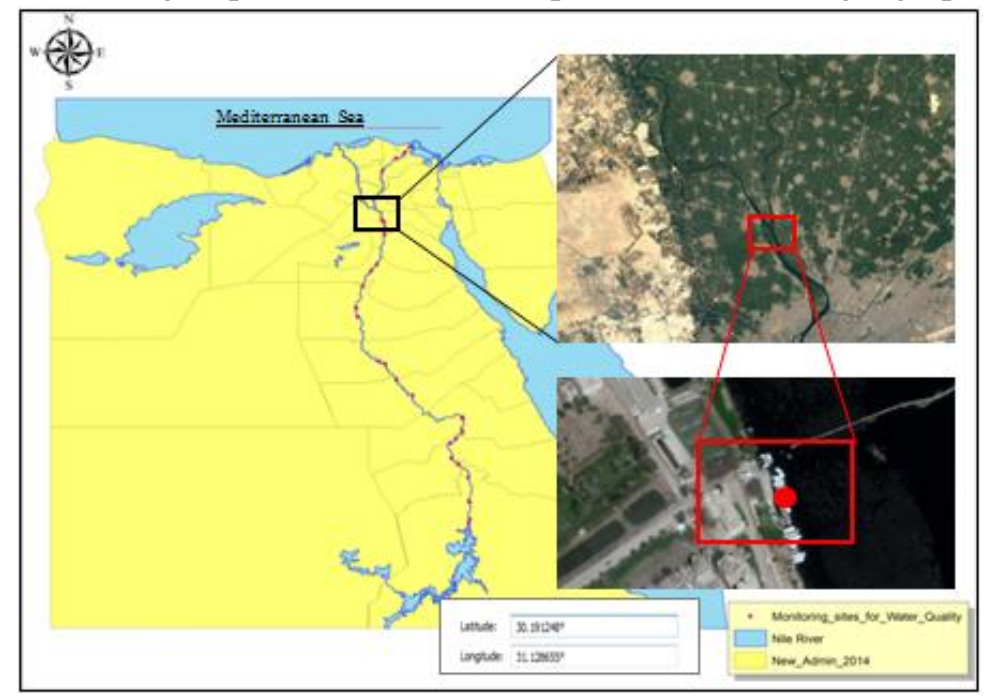

Fig. 2. Location of the pilot sensing terminal node

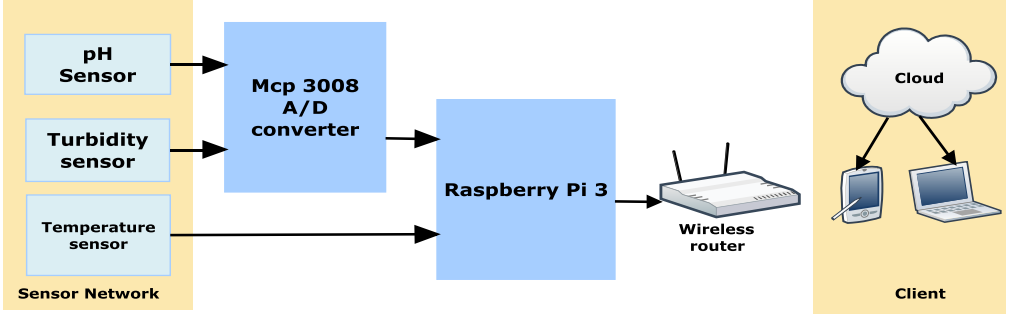

Fig. 3. Block diagram of the prototype system 
Furthermore, with the help of IoT environment and cloud computing, a facility to access data remotely from all over the world can be provided. Figure 4 shows the deployment and setup of the EWF components at the pilot sensing terminal node.

Water pollution fluctuation due to the increase in population, industrialization and other anthropological activities strongly affects the physical, chemical and biological parameters of the River Nile water. Therefore, this fluctuation needs to be instantaneously monitored to make up the sound decision at the right time. The present study demonstrates the ability of the wireless remote sensing technique to monitor water quality in the river instantaneously. The water quality status and quantification of different water quality parameters in the river are monitored using a wireless remote sensing technique. A sampling site has been selected and prepared as a case study for measuring the different parameters in Nile River.

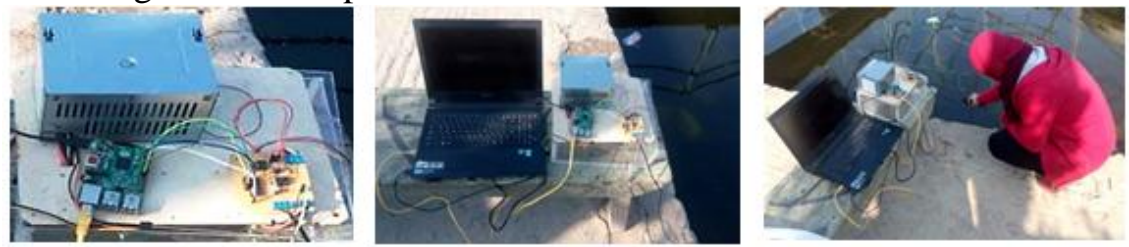

Fig. 4. Components of the EWF at the pilot sensing terminal node

\subsection{Upgrading the water quality monitoring system at NRI}

The authors developed and upgraded the current water quality monitoring system at NRI so that it can collect real-time data. The existing system depends on EXO Sond device (Figure 5). The Exo specification are depth rating of 250 meters, Internal Logging Memory Capacity 512 MB, Communications Bluetooth, Field Cable, power external 9-16 VDC, Internal 2D size batteries, battery life 90 days typically, and dimensions of diameter 4.70 $\mathrm{cm}$, Length $64.77 \mathrm{~cm}$, and Weight $1.42 \mathrm{~kg}$. The EXO collects data and stores it on board the Sond but it is not able to transmit the data over wireless network at the real time. The authors developed a new system depending on wireless connection between EXO and PCs via Bluetooth by micro-controller (Latte Panda). This system collects the measured realtime water quality data and stores it in a Master Station. The EXO Sond is a multiparameter instrument that collects water quality data. The Sond collects the data with up to four user replaceable sensors and an integral pressure transducer. Each sensor measures its parameter via a variety of electrochemical, optical, or physical detection methods. Each port accepts any EXO sensor and automatically recognizes its type. Depending on userdefined settings, the EXO collects data and stores it onboard the Sond and transfers the data to a data collection platform (DCP) or relays it directly to a user's PC or EXO Handheld. The upgraded system is shown in Figure 6 with both Kor and Team Viewer installed on both the Latte Panda and the users' PC. The user can initiate a session to get a remote access and control over Latte Panda using the Team Viewer. The Sonde is connected to Latte Panda via a Bluetooth connection. The user can start the Kor software that is installed on the Panda, get the sensors readings, save them to a file and even transfer the data files to their own PCs. Measurements of $\mathrm{pH}$, turbidity and temperature obtained by both the Prototype and EXO sensors have been compared for calibration. 


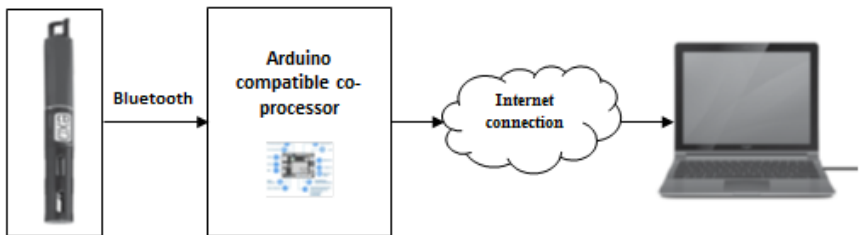

Fig. 5. Block Diagram of EXO Sonde connections at the pilot sensing terminal node
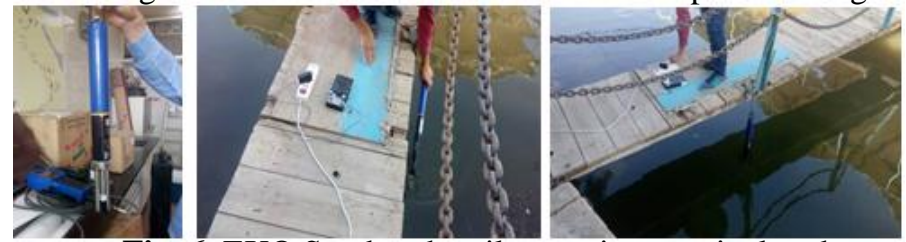

Fig. 6. EXO Sond at the pilot sensing terminal node

\subsection{Development of a dynamic website and mobile application}

The developed website is of a dynamic type. Dynamic websites usually contain web pages that are generated in real-time as shown in Figure 7. These pages include Web scripting codes as ASP. When a dynamic page is accessed, the code within the page is parsed on the Web server and the resulting HTML is sent to the client's Web browser. They are easier than static websites to maintain. This is because each static page contains unique content, meaning they must be manually opened, edited, and published whenever a change is made. In addition, the developed dynamic pages access information from the developed database. Therefore, to alter the content of a dynamic page, the webmaster may only need to update a database record.
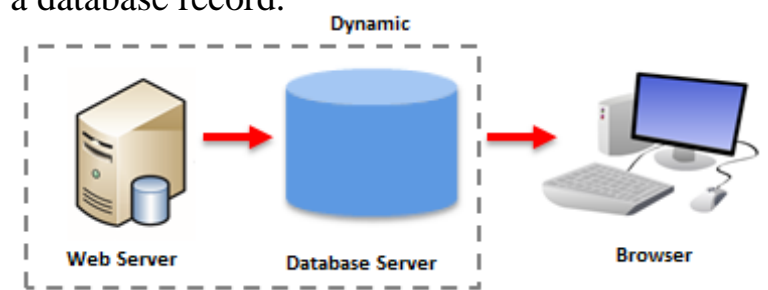

Fig. 7. dynamic website
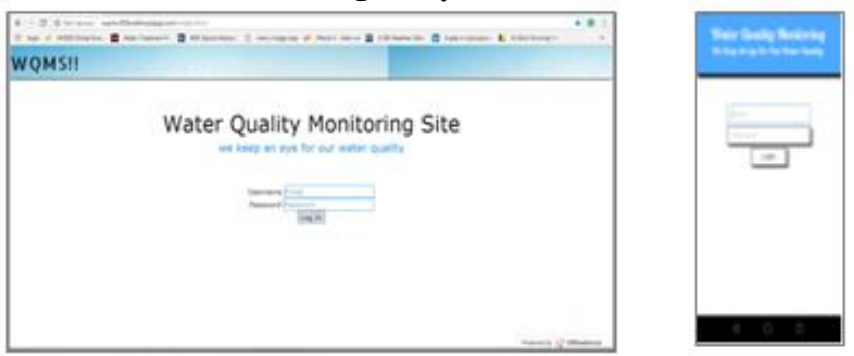

a. Web application

b. Mobile application

Fig. 8. Developed website and mobile application

\section{Results and discussion}

\subsection{Results}

The experiments setup are conducted based on the arrangements shown in Figures (3) and (5), where both systems are used to measure the water quality parameters. The 
802

JES, Assiut University, Faculty of Engineering, Vol. 47, No. 6, November 2019, pp. 795-806

following charts show the water quality parameters measurements by the developed Prototype and updated EXO sensors.

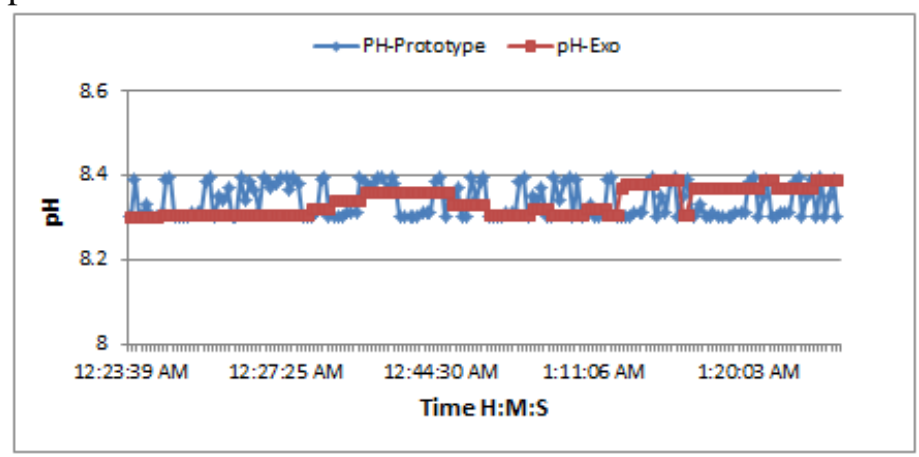

a. $\mathrm{pH}$ measurements by the Prototype and EXO sensors

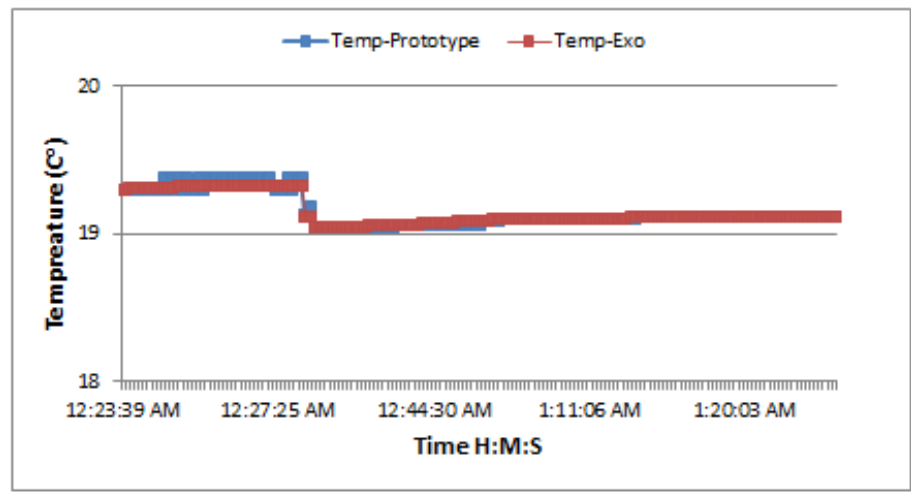

b. Temperature measurements by the Prototype and EXO sensors

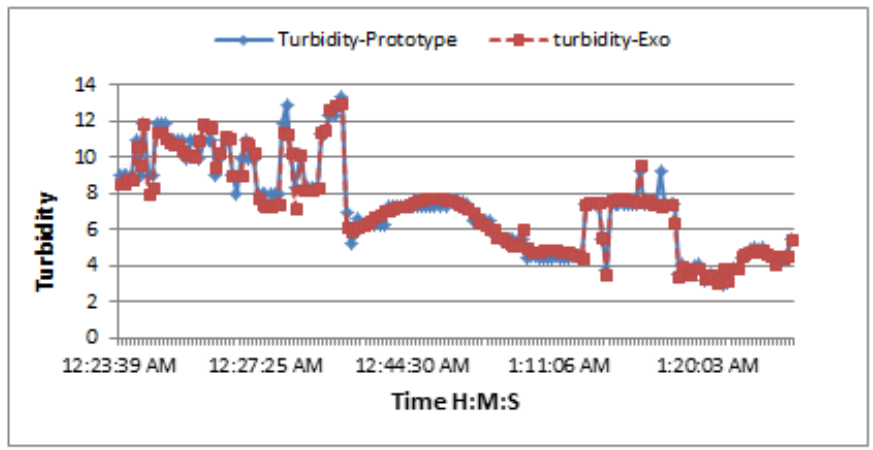

c. Turbidity measurements by the Prototype and EXO sensors

Fig. 9. Parameters measurements by the Prototype and EXO sensors

\subsection{Calibration of the prototype sensors}

The measurements of $\mathrm{pH}$, temperature, and turbidity by both; the Prototype and EXO sensors are presented in Figure 9 above. The Performance of the Prototype sensors have been evaluated statistically by calculating the Root Mean Squared Error (RMSE) as follows:

RMSE $=\sqrt{\frac{\sum\left(\mathrm{X}_{\text {Prototype }}-\mathrm{X}_{\text {Exo }}\right)}{\mathrm{N}}}$ 
Where;

$\mathrm{X}_{\text {Prototype }}=$ Values measured by the prototype sensor;

$\mathrm{X}_{\mathrm{Exo}} \quad=$ Values measured by the EXO device; and

$\mathrm{N} \quad=$ Number of measurements

The values of the RMSE show that the values of the error percentage were accepted ( 0.19 for $\mathrm{pH}, 0.056$ for temperature, and 0.52 for turbidity).

\subsection{Data analysis by statistical model Minitab16}

Statistical model Minitab16 was used for the evaluation and testing of the descriptive two systems readings. A 2-sample test was used to evaluate the effectiveness of the two devices samples. The effectiveness of these two devices were compared by determining whether or not there is any evidence that the difference between the two devices is different from zero.

Table (1) gives a confidence interval for the difference in all parameter means. For $\mathrm{pH}$, turbidity, and water temperature parameters, a $95 \%$ confidence interval includes zero. This suggests that there is no difference. The next step is the hypothesis test result. The test statistic is 0.12, with P-value of 0.904, and 301 degrees of freedom DF.

To determine whether the difference between the population means is statistically significant, compare the p-value to the significance level. Usually, a significance level (denoted as $\alpha$ or alpha) of 0.05 works well. A significance level of 0.05 indicates a $5 \%$ risk of concluding that a difference exists when there is no actual difference.

If the p-value is less than or equal to the significance level, the decision is to reject the null hypothesis. Which concluded that the difference between the population means is statistically significant. On the other hand, If the p-value is greater than the significance level, the decision is to fail to reject the null hypothesis. There is no enough evidence to conclude that the difference between the population means is statistically significant.

Since the P-value is greater than the commonly chosen 0.05 , there is no evidence for a difference in parameter values.

Figure 10 clearly shows the boxplots of $\mathrm{pH}$, temperature, and turbidity measurements by both the Prototype and EXO sensors. It has been found that there is no difference between the readings of the two devices. The readings of each parameter almost has the same mean.

Table1.

2-sample Test (test and confidence Interval)

\begin{tabular}{|c|c|c|c|c|c|}
\hline $\begin{array}{c}\text { water quality } \\
\text { parameters }\end{array}$ & Difference & $95 \%$ CI for difference & T-Value & P-Value & DF \\
\hline $\mathrm{pH}$ & -0.00047 & $(-0.00817,0.00723)$ & -0.12 & 0.904 & 301 \\
\hline Turbidity & -0.006 & $(-0.557,0.545)$ & -0.02 & 0.983 & 319 \\
\hline Temperature & 0.0028 & $(-0.0703,0.0760)$ & 0.08 & 0.939 & 319 \\
\hline
\end{tabular}




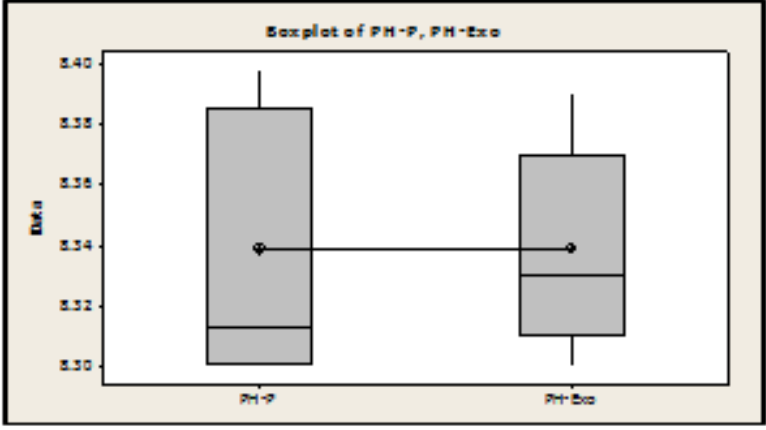

a. Boxplot of $\mathrm{pH}$ parameter (Prototype and EXO)

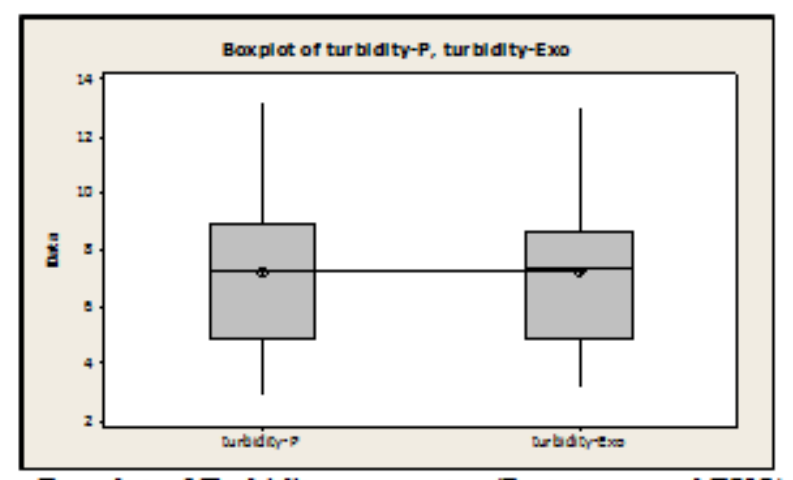

3. Boxplot of Turbidity parameter (Prototype and EXO)

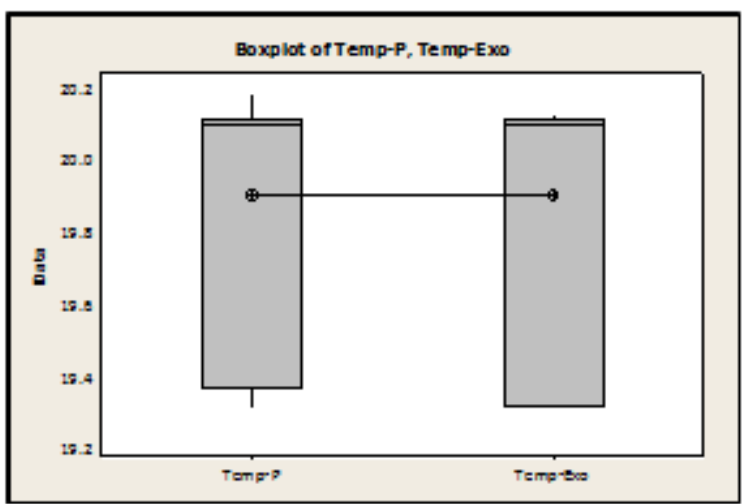

Fig. 10. Boxplot of the measurements (Prototype and EXO)

Box plots for $\mathrm{pH}$ values showed that, for prototype the variability (the box height) is greater than EXO but still have the same median.

However, for turbidity and temperature the two box plots for each parameter have the same variability (the same inter-quartile range and the same height) and the readings of the two devices for $\mathrm{pH}$, turbidity and temperature are close.

\subsection{Cost analysis}

The cost of each item and the total cost are compared for the Pilot EWF using both the Prototype and EXO devices. The overall costs of the currently developed Prototype 
Noha Kamal et al., Early Warning And Water Quality,Low-Cost Iot Based Monitoring System

including sensors, raspberry Pi and other extra costs ( wires, power supply ,...etc) have been found to be only 197 \$ whereas, they were 11,130 \$ for EXO.

Table 2.

Comparison for the costs of the Pilot EWF using both; the prototype and EXO

\begin{tabular}{|l|c|c|}
\hline Item & Prototype & EXO \\
\hline pH Sensor & 47 & \multirow{2}{*}{11,000} \\
\hline Turbidity Sensor & 59 & \\
\hline Temperature Sensor & 5 & - \\
\hline Raspberry Pi & 56 & 100 \\
\hline Latte-panda & - & 30 \\
\hline Extra & 30 & $11,130 \$$ \\
\hline Total & $197 \$$ & \\
\hline
\end{tabular}

\section{Conclusion and Recommendation}

The developed Prototype through this work represented a low-cost Early Warning Framework (EWF) for water quality monitoring of the River Nile in the real time, which supporting the decision making process. The comparison results between developed Prototype and EXO revealed that there is no significant difference between the two measurements according to a statistical analysis done using the Minitab 16 statistical model. The Root Mean Squared Error (RMSE) values showed that the error percentages were accepted for the three monitored parameters ( 0.19 for $\mathrm{pH}, 0.056$ for temperature, and 0.52 for turbidity). Moreover, the overall cost of the developed Prototype including sensors, raspberry $\mathrm{Pi}$ and all other expenses was found to be only 197 \$ as compared to 11,130 \$ when using EXO.

The developed prototype provides a low-cost and labor-saving water quality monitoring and early warning system. Once installing this system successfully at the current different water quality stations, the network is intended to be expanded and the Prototype units will be deployed at other strategic sites along the River Nile, irrigation canals, and groundwater monitoring sites. It should be emphasized that priority is given to critical sites upon further expansion.

I is strongly recommanded to develop an action plan that uses the new developed prototype in data management and reporting. Also, the derived information and knowledge should be used in integrated water resources management.

In addition, it is recommended to develop the prortotype system to be battery or solarred operated and mobile communications may be used as the locations of the water quality monitoring stations be along the River Nile including locations where no WiFi or even no electricity is available.

\section{REFERENCES}

[1] K. H. Kamaludin and W. Ismail, "Water quality monitoring with internet of things (IoT)," 2017 IEEE Conference on Systems, Process and Control (ICSPC), Malacca, 2017, pp. 18-23.doi: 10.1109/SPC.2017.8313015.

[2] O. Adedokun O.A.and Adeyemo, "Limnological variation and nutrient load of the river system in ibadan metropolis, nigeria," European journal of scientific research, vol. 23, pp. $98-108,2008$. 
[3] Majid Bayani, Alberto Segura, Marjorie Alvarado and Mayra Loaiza,” IoT-Based Library Automation and Monitoring system: Developing an Implementation framework of Implementation", e-Ciencias de la Información, volume 8, number 1, Jan-Jun 2017 ISSN: 1649-4142. DOI: https://doi.org/10.15517/eci.v8i1.30010.

[4] H. Chengfang, X. Xiao, S. Dingtao, C. Bo, and W. Xiongfei, "Study of water pollution early warning framework based on internet of things," International Archives of the Photogrammetry, Remote Sensing and Spatial Information Sciences, vol. XLI-B8, no. 5, pp. 335-338, 2016.

[5] A. N. Prasad, K. A. Mamun, F. R. Islam, and H. Haqva, "Smart water quality monitoring system," in 2015 2nd Asia-Pacific World Congress on Computer Science and Engineering (APWC on CSE), pp. 1-6, Dec 2015.

[6] G. S. Menon, M. V. Ramesh and P. Divya, "A low cost wireless sensor network for water quality monitoring in natural water bodies," 2017 IEEE Global Humanitarian Technology Conference (GHTC), San Jose, CA, 2017, pp. $1-8$. doi: 10.1109/GHTC.2017.8239341.

[7] K. Thiyagarajan, S. Pappu, P. Vudatha, and N. , "Intelligent iot based water quality monitoring system," International Journal of Applied Engineering Research, vol. 12, p. 5447, 102017.

[8] B. Das and P. C. Jain, "Real-time water quality monitoring system using Internet of Things," 2017 International Conference on Computer, Communications and Electronics (Comptelix),Jaipur,2017, pp.78-82. doi: 10.1109/COMPTELIX. 2017.8003942.

[9] F. Wang, L. Hu, J. Zhou, and K. Zhao, "A survey from the perspective of evolutionary process in the internet of things," International Journal of Distributed Sensor Networks, vol. 11, no. 3, p. 462752, 2015.

\section{نظام إنذار مبكر منخفض التكلفة لرصد نوعية المياه قائم على إنترنت الأشياء}

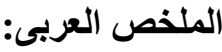

يشتمل برنامج رصد ومر اقبة نوعية المياه الحالي في مصر علي اجر اء الكثير من القياسات الفيزيائية والكيميائية

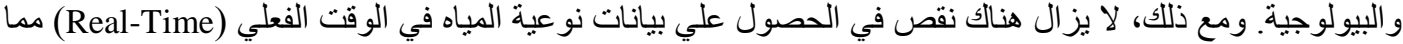

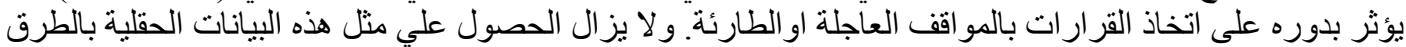

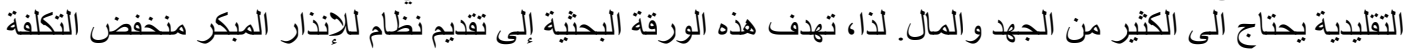
وموفر للايدى العاملة لرصد نوعية مباه نهر النيل وقائم على إنترنت الأشياء.

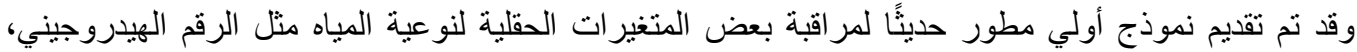

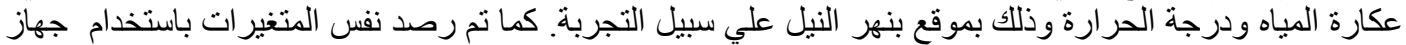

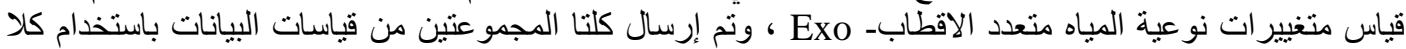

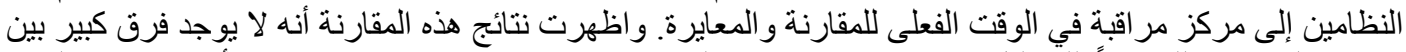

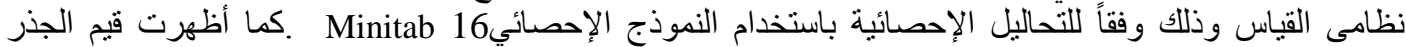

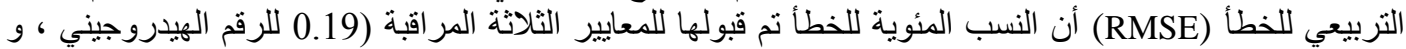

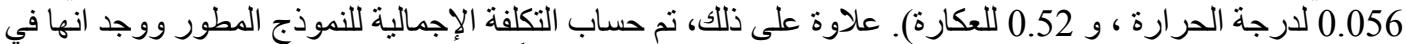

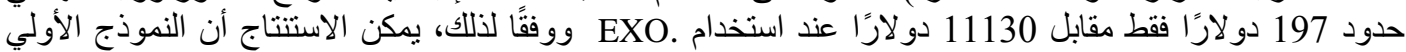

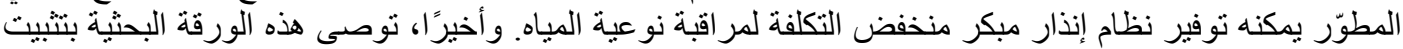

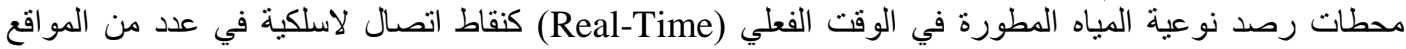
الاستر اتيجية على طول نهر النيل داخل مصرة في الهر. 